\title{
The Use of Continuous Silicone Oil Infusion as a Peroperative Tool to Facilitate Break Localisation, Vitreous Base Dissection and Drainage of Subretinal Fluid
}

\author{
David Wong Wico Lai Wadid Yusof \\ Eye Institute, LKS Faculty of Medicine, University of Hong Kong, Hong Kong, SAR, China
}

\section{Key Words}

Silicone oil infusion • Retinal break • Vitreous base shaving • Subretinal fluid drainage

\begin{abstract}
Introduction: We introduce the use of low-molecular-weight silicone oil as an infusion and as a peroperative tool to facilitate three specific surgical steps, namely internal search for retinal breaks, vitreous base shaving and drainage of subretinal fluid. Method and Patients: Ethics committee approval was obtained to test this novel agent in a small number of cases as a pilot study. Following vitrectomy the posterior retina was reattached and stabilised using perfluorocarbon liquid (PFCL). The infusion of balanced salt solution was then stopped and replaced with $5 \mathrm{mPas}$ silicone oil. Subsequent surgery was assessed as to whether the infusion was helpful in the three surgical steps. Five cases were selected for this pilot study. Three presented with long-standing rhegmatogenous retinal detachment (RRD) of 2 or more months: 1 had dense vitreous haemorrhage and no fundal view at presentation; 2 had proliferative vitreoretinopathy. Of the remaining 2 cases, 1 had a giant retinal break and the other case had a combined diabetic tractional retinal detachment and RRD. Results: Additional retinal breaks were
\end{abstract}

found in 2 patients. The silicone oil joined with the PFCL to form a single bubble. The vitreous base along with the operculum of any retinal break was reflected anteriorly. The infusion of oil stabilised the anterior retina, making it possible to shave the vitreous closely in all 5 patients. Visualisation of the vitreous gel was also improved. There was a clear interface between vitreous and oil because of the large difference between the refractive indices of vitreous and oil. Four of the 5 patients had a total reattachment with one procedure. In the 2 cases in which 5,000 mPas silicone oil was used for longterm postoperative tamponade, no emulsification was observed. Follow-up of patients ranged from 6 to 13 months. There were no additional serious adverse reactions recorded. Conclusion: We found that the experience of using silicone oil was very highly positive.

Copyright $\odot 2011$ S. Karger AG, Basel

\section{Introduction}

Successful repair of rhegmatogenous retinal detachment (RRD) crucially depends on the identification and closure of all causative retinal breaks. It has been shown that with meticulous internal search and peroperative sealing of retinal breaks, a high rate of success could be

\section{KARGER \\ Fax +41613061234 \\ E-Mail karger@karger.ch}

www.karger.com
(C) 2011 S. Karger AG, Basel

$0030-3755 / 11 / 2265-0053 \$ 38.00 / 0$

Accessible online at:

www.karger.com/oph
David Wong

Eye Institute, LKS Faculty of Medicine, University of Hong Kong

Cyberport 4

Hong Kong, SAR (China)

E-Mail shdwong@hku.hk 
achieved with vitrectomy surgery even without using postoperative tamponade [1]. Equally, a common cause of failure to repair retinal detachment is proliferative vitreoretinopathy (PVR) [2, 3]. Long-term tamponade is required and silicone oil is the choice for many. In the past, we and others have argued that effective tamponade with silicone oil depends on achieving a near total fill $[4,5]$. This in turn relies on complete drainage of subretinal fluid (SRF) and close shaving of the vitreous base. An intraoperative tool that could facilitate these three important procedures - namely exhaustive internal search for retinal breaks, close vitreous base shaving and complete drainage of SRF - would be highly relevant.

Silicone oil is a polymer of dimethylsiloxane. The higher the molecular weight, the greater the shear viscosity of the liquid. Conversely, when the molecular weight is sufficiently low, it is possible to produce a fluid that is water-like. We have chosen to use a silicone with a shear viscosity of $5 \mathrm{mPas}$. This was designed as a peroperative tool to be used as an infusion in place of balanced salt solution. The oil was supplied in bottles of $500 \mathrm{ml}$ in volume (Fluoron GmbH, Ulm, Germany). Chemically, it is the same as conventional silicone oil 1,000 mPas or 5,000 $\mathrm{mPas}$. The novelty is its low viscosity and the way it is used. The other physical properties remain unchanged: the specific gravity is $0.97 \mathrm{~g} / \mathrm{ml}$ and the refractive index is 1.40 .

\section{Patients and Method}

We obtained ethical approval to test silicone oil infusion on a small number of patients. In obtaining consent, the patient understood that the use of silicone infusion as an operative adjunct was novel, that we would only decide peroperatively to proceed to use the infusion if we were convinced it could be useful, that extra tests and clinic visits might be necessary and that we would be monitoring all serious adverse effects. The eligibility criteria were deliberately inclusive for this pilot study. All patients with RRD were considered eligible, provided they were able to give informed consent. We excluded patients with breaks in fixed retinae because there was a chance of subretinal migration of silicone.

A single surgeon (D.W.) performed all the surgeries. Posterior vitrectomy was carried out and triamcinolone was used to identify and remove any residual vitreous cortex. The posterior retina was then stabilised with the use of perfluorocarbon liquid (PFCL). Vitreous base shaving was performed. The infusion of balanced salt solution was stopped and the infusion of low-molecularweight silicone oil commenced. Vitreous base shaving, internal search and drainage of SRF through existing peripheral retinal breaks were carried out. Endolaser was used for retinopexy, after which the PFCL was evacuated by passive suction. An air/silicone oil exchange was then carried out. Care was taken to remove the last drop of the low-molecular-weight silicone oil. Finally, either
$\mathrm{C}_{3} \mathrm{~F}_{8}$ gas or conventional 5,000 mPas silicone oil was injected into the air-filled eye as a postoperative endotamponade. The surgeon rated the usefulness of the silicone oil infusion in each of the three surgical steps.

\section{Results}

Seven patients were included and consented to the study. At the time of surgery, we did not proceed in 2 cases because we were highly confident that we had found all retinal breaks, that there was no significant PVR and that all surgical steps could be easily achieved without the use of silicone oil infusion.

We used the silicone oil infusion in 5 patients. Three presented with long-standing retinal detachments. One of the 3 had a dense vitreous haemorrhage with no fundal view, another case had posterior PVR, and the last case had both anterior and posterior PVR combined with limited posterior vitreous detachment. Of the remaining 2 cases, 1 had a combined diabetic tractional retinal detachment and RRD, and the other had a giant retinal break. The surgery and clinical details as well as the PVR grades are summarised in table 1 . The usefulness of the infusion as rated by the surgeon is summarised in table 2 .

\section{Shaving of Vitreous Base}

Silicone and PFCL are both hydrophobic. Once in contact with one another, the two liquids joined to form a single bubble. This combined bubble was a heavier-thanwater bubble (as expected). Any aqueous or residual vitreous gel was displaced both anteriorly and laterally.

In all instances, vitreous base shaving was carried out using just PFCL, before the silicone oil infusion. After the infusion, in all 5 cases, it was possible to shave the vitreous closer. In all cases, the anterior retina was stabilised, even though there might still have been residual SRF underneath the retina. The vitreous cutter could therefore work much closer to the retina without fear of aspirating the retina or causing iatrogenic holes. In all cases, the gel was highlighted. The refractive index of the silicone oil infusion was 1.40. The refractive index of the vitreous gel was around 1.34. The difference was such that the vitreous gel could clearly be seen. The end point for vitreous shaving was therefore definite. We continued until only the thinnest layer of gel remained. In the one patient with combined diabetic tractional retinal detachment and RRD, it was also possible to cut away all the blood in the vitreous base and to remove the anterior hyaloid. The surgeon rated the silicone oil as being helpful in all 5 cases. 


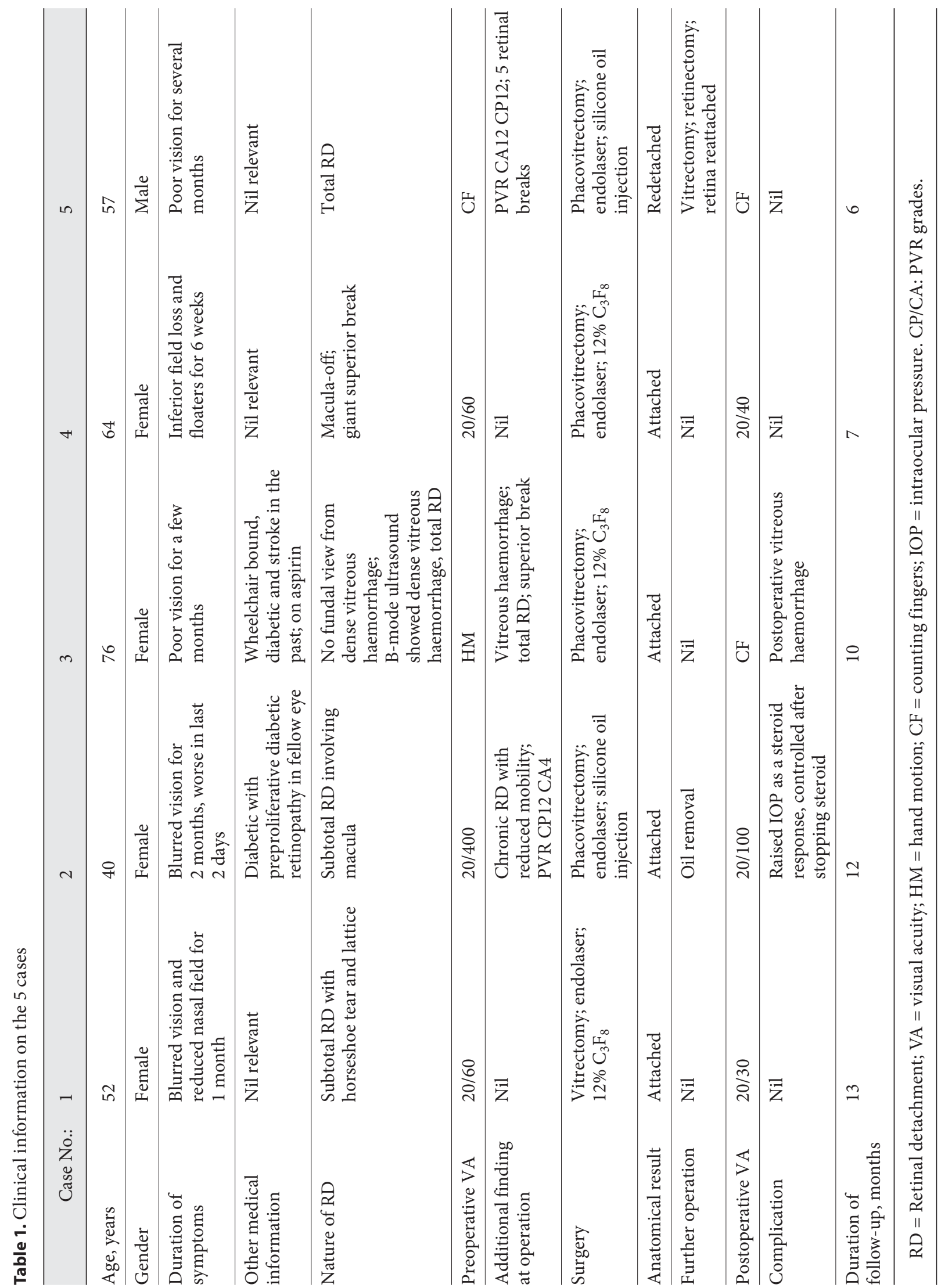


Table 2. Surgeon's rating on the usefulness of silicone oil infusion

\begin{tabular}{llllll}
\hline \multicolumn{1}{c}{ Case No.: } & 1 & 2 & 3 & 4 & 5 \\
\hline $\begin{array}{l}\text { Shaving of vitreous base } \\
\text { Internal search }\end{array}$ & Helpful & Helpful & Helpful & Helpful & Helpful \\
Drainage of SRF & Not helpful & Not helpful & Not helpful & $\begin{array}{l}\text { Helpful because } \\
\text { additional breaks found } \\
\text { Helpful: SRF drained } \\
\text { completely }\end{array}$ & $\begin{array}{l}\text { Helpful because } \\
\text { additional breaks found } \\
\text { Not helpful }\end{array}$ \\
\hline
\end{tabular}

\section{Internal Search for Retinal Breaks}

This was rated as helpful in 2 of the 5 cases because additional retinal breaks were found. Silicone oil infusion and PFCL together formed a combined bubble that was heavier than water. The vitreous and the operculum of $U$ tears were reflected anteriorly (fig. 1). The posterior border of the vitreous base was clearly seen inside the silicone oil infusion. This was because of the high refractive index of oil (1.40 as opposed to 1.29 for $n$-octane and 1.31 for perfluorodecalin). The internal search was therefore greatly facilitated. Even where no retinal breaks were found, the clear delineation of the posterior border of the vitreous base (fig. 1) helped us to place the encircling endolaser more accurately in the 2 cases with PVR.

\section{Drainage of SRF}

In all cases, PFCL was used. Any SRF was displaced anteriorly. When we infused the silicone oil, the peripheral retina appeared to be flattened. We performed drainage through peripheral retinal breaks to evacuate any remaining SRF. In 2 patients, the SRF drainage was seemingly complete. Only when PFCL was removed did it then become obvious that there still was residual SRF. The SRF was displaced backwards towards the posterior pole by the incoming silicone oil bubble. In this respect, the silicone oil infusion was not that different from air infusion. Complete drainage of SRF therefore remained difficult. It was achieved only in 1 case: that of the giant retinal tear. In 4 of the 5 cases, the silicone oil was not particularly helpful. However, it was noted that visualisation through the oil was excellent. There was no problem with condensation, which could hamper the view in pseudophakic eyes. There also was no feathery lens opacity observed in the phakic patient. In this regard, silicone oil performed better than air.

\section{Adverse Effect}

In the 2 patients in whom long-term postoperative silicone oil tamponade was used, no emulsification was ob-

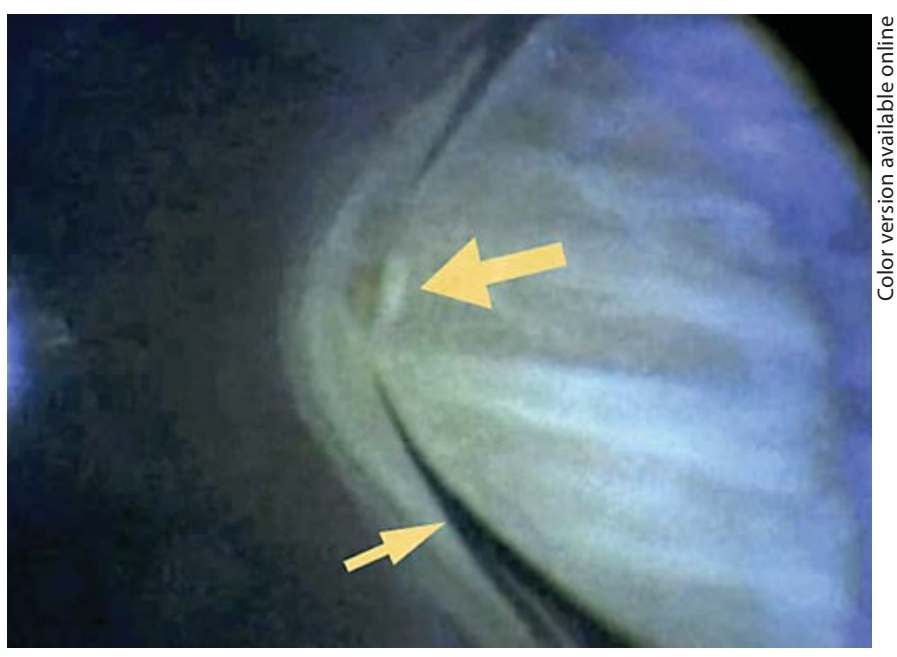

Fig. 1. Peroperative photograph showing silicone oil infusion in situ. The posterior border is seen as a sharp line (small arrow). The operculum of a small retinal break is reflected anteriorly (large arrow).

served. There was 1 patient with raised intraocular pressure. That was attributable to steroid response. The intraocular pressure subsided when the topical steroid was stopped. One patient with PVR needed one further surgery. One patient on aspirin who presented with preoperative haemorrhage experienced a postoperative haemorrhage.

It was noted that at $5 \mathrm{mPas}$, the silicone oil was still several time more viscous than water, which at body temperature had a viscosity of around $0.8 \mathrm{mPas}$. This was reflected in the flow rate. On occasions when oil was infused at the same time as vitreous fluid was aspirated, the intraocular pressure could drop because the inflow was not keeping up with the outflow. Once this was appreciated, this complication could be avoided. 


\section{Discussion}

In the UK, John Scott pioneered vitrectomy and silicone oil injection for complex retinal detachment [6]. His early surgeries did not involve using any saline infusion. The silicone oil was injected incrementally whilst he removed the vitreous gel from around the oil bubble and drained SRF to create space. It was impressive how thorough the vitrectomy was using silicone oil. The majority of this kind of surgery was carried out using indirect ophthalmoscopy. We were also impressed how well the vitreous gel could be visualised because of the difference between the refractive indices of vitreous and oil. As there was no saline infusion, intraocular pressure maintenance was challenging. This was achieved using digital pressure on the eye during vitrectomy and in between injections of the oil.

We had the idea of using low-molecular-weight silicone oil. At $5 \mathrm{mPas}$, the oil could be infused and intraocular pressure could be maintained. The main motivation for introducing this as an adjunct to vitrectomy was to see if we could perform the three important surgical steps better, namely thorough shaving of the vitreous base, exhaustive identification of all retinal breaks and complete drainage of SRF.

The slightly higher refractive index meant that there was a very clear interface between the vitreous gel and oil. The incoming oil infusion joined with the PFCL to form a single bubble such that any aqueous would be displaced laterally and also anteriorly. The anterior displacement of the gel was anticipated because the combined bubble would have a specific gravity higher than water. The use of oil infusion had the excellent effect of lifting the gel base, which greatly assisted the internal search for retinal breaks. We were impressed that any operculum was always reflected anteriorly (fig. 1).

We were very conscious that leaving low-molecularsilicone oil might admix with conventional silicone oil. Such a low-molecular-weight component could promote emulsification [7]. It was necessary in all instances to go through an air/silicone infusion exchange to make sure that the very last droplet of oil was removed. Infusing silicone oil under the retina was also a concern. We were careful not to select cases with retinal breaks in fixed retinae. Before infusion of oil was used, sufficient peeling had to be done to ensure that the retina was reasonably mobile.

Overall, we were greatly encouraged by the performance of the infusion. We concluded that silicone infusion was very helpful for break detection and vitreous base shaving. The indication for its use could therefore include cases where no peripheral retinal breaks were identified during the internal search. In cases of PVR where vitreous base dissection was indicated, the infusion offered the distinct advantage of keeping the retina stable and offering excellent visualisation. Kirchhof had used the infusion for managing severe trauma and haemophthalmos. The silicone oil infusion maintained a clear medium. A video recording of this can be seen online [8].

\section{Disclosure Statement}

Prof. D.W. has filed a preliminary patent for the use of lowmolecular-weight silicone oil infusion as an intraoperative tool and surgical adjunct.

\section{References}

-1 Martínez-Castillo V, Zapata MA, Boixadera A, Fonollosa A, García-Arumí J: Pars plana vitrectomy, laser retinopexy, and aqueous tamponade for pseudophakic rhegmatogenous retinal detachment. Ophthalmology 2007;114:297-302.

-2 Asaria RH, Kon CH, Bunce C, Charteris DG, Wong D, Luthert PJ, Khaw PT, Aylward GW: How to predict proliferative vitreoretinopathy: a prospective study. Ophthalmology 2001;108:1184-1186.
3 Wickham L, Bunce C, Wong D, Charteris DG: Retinal detachment repair by vitrectomy: simplified formulae to estimate the risk of failure. Br J Ophthalmol 2011, E-pub ahead of print.

4 Petersen J: The physical and surgical aspects of silicone oil in the vitreous cavity. Graefes Arch Clin Exp Ophthalmol 1987;225:452456.

5 Fawcett IM, Williams RL, Wong D: Contact angles of substances used for internal tamponade in retinal detachment surgery. Graefes Arch Clin Exp Ophthalmol 1994;232: 438-444.

\footnotetext{
6 Scott JD: The treatment of massive vitreous retraction by the separation of pre-retinal membranes using liquid silicone. Mod Probl Ophthalmol 1975;15:185-190.

$\checkmark 7$ Heidenkummer HP, Kampik A, Thierfelder S: Emulsification of silicone oils with specific physicochemical characteristics. Graefes Arch Clin Exp Ophthalmol 1991;229:88-94.

8 http://www.eyemoviepedia.com/videos/2835569646/147.
} 